ISSN 1027-5495. Functional Materials, 24, No.4 (2017), p. 584-592

doi:https://doi.org/10.15407/fm24.04.584

(C) 2017 - STC "Institute for Single Crystals"

\title{
Experimental research on mechanical properties of desert sand steel-PVA fiber engineered cementitious composites
}

\author{
Che Jialing ${ }^{1}$, Li Quanwei ${ }^{1}$, Lee Minggin', Wang Dan ${ }^{1}$ \\ ${ }^{1}$ School of Civil Engineering and Hyd raulic Engineering, Ningxia \\ University, Yinchuan Ningxia, 750021, P.R.China \\ ${ }^{2}$ Department of Civil Engineering, Chaoyang University of Technology, \\ Taichung County, 41349, Taiwan
}

Received August 23,2017

\begin{abstract}
An orthogonal experimental design method involving five-factor and four-level is adopted for the mix design of Desert Sand Steel-PVA fiber ECC. The effect of each level on Mechanical properties of ECC and the difference of Mechanical properties between each level is analyzed. The influence of different experimental factors is discussed, which includes water-binder ratio, fly ash substitution rate, desert sand substitution rate, proportion of PVA fiber and proportion of steel fiber. The experimental results indicate that water-binder ratio and fly ash substitution rate are the most principal and significant influencing factors on the compressive strength of ECC, regardless of age. Steel fiber is conducive to development of splitting tensile strength; PVA fiber is conducive to the development of flexural strength. High strength ECC can be prepared when the desert sand substitution rate is high. As the raw material of ECC, river sand can be $90 \%$ replaced by desert sand.
\end{abstract}

Keywords: Desert sand, Engineered cementitious composites(ECC), Mechanical properties, Orthogonal experiment, PVA fiber, Steel fiber.

Исследуется влияние различных фракторов на свойства экологически чистого и экономически выгодного конструкционного строительного композитного материала (ККМ). Исследовалась смесь волоконной эмульсии Desert Sand Steel-PVA. Для исследований использовался метод ортогонального экспериментального проектирования с пятифакторным (соотношение вода-связующее, скорость замещения летучей золы, скорость замещения песка пустыни, доли волокна ПВА и доли стального волокна) и четырехуровневым испытанием на прочность. Экспериментальные результаты показывают, что соотношение воды и связующего материала, а также скорость замещивания летучей золы являются наиболее влияющими факторами на прочность на сжатие ККМ, независимо от срока его получения. Стальное волокно способствует развитию растягивающей силы расщепления; ПВА волокно способствует развитию прочности на изгиб. Высокопрочный ЕCС может быть приготовлен, при большой скорости замещения песка пустынию. В качестве сырья ККМ речной песок может быть заменен на 90\% пустынным песком .

Експериментальні дослідження механічних властивостей конструкційного композитного матеріалу Desert Sand Steel-PVA. Che Jialing, Li Quanwei, Lee Minggin, Wang Dan.

Досліджуеться вплив різних чинників на властивості екологічно чистого та економічно вигідного конструкційного будівельного композитного матеріалу (ККП). Досліджувалася суміш волоконної емульсії Desert Sand Steel-PVA. Для досліджень використовувався метод ортогонального експериментального проектування з п'ятифакторна (співвідношення вода- 
сполучна, швидкість заміщення летючого попелу, швидкість заміщення піску пустелі, частки волокна ПВА і частки сталевого волокна) і чотирьохрівневим випробуванням на міцність. Експериментальні результати показують, що співвідношення води і сполучного матеріалу, а також швидкість замещіванія летючої золи є найбільш впливають факторами на міцність на стиск ККМ, незалежно від терміну його отримання. Сталеве волокно сприяе розвитку сили, що розтягує розщеплення; ПВА волокно сприяе розвитку міцності на вигин. Високоміцний ECC може бути приготований, при великій швидкості заміщення піску пустелі. В якості сировини ККМ річковий пісок може бути замінений на 90\% пустельним піском.

\section{Introduction}

Engineered Cementitious Composites (hereinafter referred to as ECC) was proposed in the last late century ECC is micromechanically designed composites with fiber as reinforcement material, cement as main base material. It features outstanding energy consuming capacity, strain hardening and crack steady state development. ECC can effectively improve the seismic resistance of the structure [1], extend service life of the material [2].

Research on steel-PVA cementitious composites show that addition of fly ash, increase of fiber proportion help multiple cracking [3.4]. PVA-ECC has outstanding property of peak delay [5]. The damage crack of PVA-ECC is less wide, which can be controlled within 100um [2, 6], Harbin Institute of Technology researched high fly ash proportion ECC, its 28d limit tensile strain is all above $3 \%$, exhibiting outstanding strain-hardening property [7]. From the angle of sustainability of the environment, ECC consumes less resource, emits less pollution [8]. Adoption of ECC can increase compressive strength and tensile strength of the concrete test piece [9]. Experiments show that compressive strength of 4-day aged ECC can meet the strength required by deck slab design, conducive to speeding up construction [10]. Using micromechanical design can achieve ideal performance of ECC [11]. Fly ash, water-binder ratio, fiber disperse condition all can influence tensile property of ECC [12, 13]. When steel fiber proportion is $2.0 \%$, difficult dispersal and serious caking of steel fiber results strength of ECC lower than that of the test piece with steel fiber proportion $1,5 \%[14]$.

Large amount of non-renewable building material is consumed, so using a substitute for building material is of significance for sustainable development of the industry. Sand for building has limited reserve and cannot be regenerated within short term worldwide, while every continent has deserts of different size, and desert sand can partly substitute for river sand used for concrete, whose smaller grain size makes concrete inside more even, more compact. Currently, the thermal power is still the main electric source globally, nowhere can place a large amount of waste fly ash discharged from thermal power plants. Fly ash can substitute for cement to a certain extent, and improve performance of concrete, avoid waste pollution. Desert sand and fly ash are used in concrete as substitute material, saving resources and lowering cost. Desert sand steelPVA fiber ECC prepared in this paper uses desert sand to substitute for part of river sand, fly ash to substitute for part of cement.

\section{Experiment design}

\subsection{Experimental material}

Saima branded p.o.42.5 ordinary portland cement of Ningxia, China, its specific surface area is $339 \mathrm{~m}^{2} / \mathrm{kg}$, the chemical composition is shown in Table 1 , the properties is shown in Table 2; for class I fly ash of Ningxia Lingwu Thermal Power Plant, water content is $0.4 \%$, water demand ratio is $90 \%$, fineness is $8.4 \%$, loss on ignition is $3 \%$, the chemical composition is shown in Table 1; the desert sand is from Tengger Desert, with average grain size $0.23 \mathrm{~mm}$, fineness modulus is 0.7 ; for sieved river sand, max grain size is $1.18 \mathrm{~mm}$, fineness modulus is 2.1; PVA fiber is produced by Japan KURARAY, steel fiber selects copper plated micro wire steel fiber produced by Hengshui Fangde Silk Screen Products Factory, performance of fiber is shown in Table 3. Admixture is powdered high efficiency polycarboxylic acid water reducer, with water reducing rate $25 \% \sim 30 \%$.

\subsection{Proportion of experiment}

The paper uses 5-factor 4-level $\left(\mathrm{L}_{16} 4^{5}\right)$ orthogonal experiment, factors and levels is shown in Table 4.

Mix design of orthogonal experiment is shown in Table 5.

\subsection{Test piece preparation and experi- mental method}

In order to make PVA fiber and steel fibers disperse evenly without caking, steel fiber premixing and PVA fiber after-mixing were adopted. First mix and agitate river sand and desert sand for 1min, add cement and fly ash agitating for $1 \mathrm{~min}$, then add steel fiber agitating for $1 \mathrm{~min}$, add water and water reducer agitating for $2 \mathrm{~min}$, finally add PVA fiber agitating for $2 \mathrm{~min}$. After agitation cast a test piece and vibrate it 
Che Jialing et al. / Experimental research on mechanical properties ...

Table 1. Chemical composition of ordinary portland cement and fly ash

\begin{tabular}{|c|c|c|c|c|c|c|c|c|c|}
\hline $\begin{array}{c}\text { Chemical } \\
\text { composition/\% }\end{array}$ & $\mathrm{CaO}$ & $\mathrm{SiO}_{2}$ & $\mathrm{Al}_{2} \mathrm{O}_{3}$ & $\mathrm{Fe}_{2} \mathrm{O}_{3}$ & $\mathrm{SO}_{3}$ & $\mathrm{MgO}$ & $\mathrm{Na}_{2} \mathrm{O}$ & $\mathrm{K}_{2} \mathrm{O}$ & Others \\
\hline Cement & 60.24 & 21.22 & 5.05 & 3.26 & 2.67 & 0.97 & 0.73 & 0.50 & 5.36 \\
\hline Fly ash & 3.16 & 53.80 & 24.60 & 9.32 & 0.42 & 1.52 & 0.28 & 0.82 & 6.08 \\
\hline
\end{tabular}

Table 2. Properties of ordinary portland cement

\begin{tabular}{|c|c|c|c|c|c|c|}
\hline Properties & $\begin{array}{c}\text { Fineness } \\
/ \%\end{array}$ & $\begin{array}{c}\text { Consistency water } \\
\text { consumption /\% }\end{array}$ & $\begin{array}{c}\text { initial setting } \\
\text { time /min }\end{array}$ & $\begin{array}{c}\text { Final set- } \\
\text { ting time } \\
/ \text { min }\end{array}$ & $\begin{array}{c}\text { Flexural } \\
\text { strength } \\
/ \mathrm{MPa}\end{array}$ & $\begin{array}{c}\text { compressive } \\
\text { strength /MPa }\end{array}$ \\
\hline Value & 4.4 & 26 & 130 & 180 & 9.0 & 55.6 \\
\hline
\end{tabular}

Table 3. Performance of experimental fiber

\begin{tabular}{|c|c|c|c|c|c||}
\hline Type & Length $/ \mathrm{mm}$ & Color & Diameter $/ \mathrm{mm}$ & $\begin{array}{c}\text { Tensile } \\
\text { strength/MPa }\end{array}$ & $\begin{array}{c}\text { Modules of } \\
\text { elasticity/GPa }\end{array}$ \\
\hline PVA fiber & 12 & white & 0.04 & 1560 & 41 \\
\hline Steel fiber & 13 & yellow & 0.2 & $>2850$ & 210 \\
\hline
\end{tabular}

Table 4. Factors and levels

\begin{tabular}{|c|c|c|c|c|c|}
\hline Factors & $\begin{array}{c}\text { Water-binder } \\
\text { ratio }\end{array}$ & $\begin{array}{c}\text { Fly ash substitute } \\
\text { rate } / \%\end{array}$ & $\begin{array}{c}\text { Desert sand } \\
\text { substitute rate/\% }\end{array}$ & $\begin{array}{c}\text { Proportion of pva } \\
\text { fiber/\% }\end{array}$ & $\begin{array}{c}\text { Proportion of } \\
\text { steel fiber/\% }\end{array}$ \\
\hline 1 & 0.19 & 15 & 0 & 0 & 0 \\
\hline 2 & 0.24 & 30 & 30 & 0.4 & 0.4 \\
\hline 3 & 0.29 & 45 & 60 & 0.8 & 0.8 \\
\hline 4 & 0.34 & 60 & 90 & 1.2 & 1.2 \\
\hline
\end{tabular}

Table 5. Mix design of orthogonal experiment

\begin{tabular}{|c|c|c|c|c|c|}
\hline $\begin{array}{l}\text { Factors } \\
\text { of specimens }\end{array}$ & $\begin{array}{c}\text { A. } \\
\text { Water- } \\
\text { binder } \\
\text { ratio }\end{array}$ & $\begin{array}{c}\text { B. } \\
\text { Fly ash sub- } \\
\text { stitute rate/\% }\end{array}$ & $\begin{array}{c}\text { C. } \\
\text { Desert sand } \\
\text { substitute } \\
\text { rate/\% }\end{array}$ & $\begin{array}{c}\text { D. } \\
\text { Proportion of } \\
\text { pva fiber } \%\end{array}$ & $\begin{array}{c}\text { E. } \\
\text { Proportion of } \\
\text { steel fiber } \%\end{array}$ \\
\hline 1 & $1(0.19)$ & $1(15)$ & $1(0)$ & $1(0)$ & $1(0)$ \\
\hline 2 & $1(0.19)$ & $2(30)$ & $2(30)$ & $2(0.4)$ & $2(0.4)$ \\
\hline 3 & $1(0.19)$ & $3(45)$ & $3(60)$ & $3(0.8)$ & $3(0.8)$ \\
\hline 4 & $1(0.19)$ & $4(60)$ & $4(90)$ & $4(1.2)$ & $4(1.2)$ \\
\hline 5 & $2(0.24)$ & $1(15)$ & $2(30)$ & $3(0.8)$ & $4(1.2)$ \\
\hline 6 & $2(0.24)$ & $2(30)$ & $1(0)$ & $4(1.2)$ & $3(0.8)$ \\
\hline 7 & $2(0.24)$ & $3(45)$ & $4(90)$ & $1(0)$ & $2(0.4)$ \\
\hline 8 & $2(0.24)$ & $4(60)$ & $3(60)$ & $2(0.4)$ & $1(0)$ \\
\hline 9 & $3(0.29)$ & $1(15)$ & $3(60)$ & $4(1.2)$ & $2(0.4)$ \\
\hline 10 & $3(0.29)$ & $2(30)$ & $4(90)$ & $3(0.8)$ & $1(0)$ \\
\hline 11 & $3(0.29)$ & $3(45)$ & $1(0)$ & $2(0.4)$ & $4(1.2)$ \\
\hline 12 & $3(0.29)$ & $4(60)$ & $2(30)$ & $1(0)$ & $3(0.8)$ \\
\hline 13 & $4(0.34)$ & $1(15)$ & $4(90)$ & $2(0.4)$ & $3(0.8)$ \\
\hline 14 & $4(0.34)$ & $2(30)$ & $3(60)$ & $1(0)$ & $4(1.2)$ \\
\hline 15 & $4(0.34)$ & $3(45)$ & $2(30)$ & $4(1.2)$ & $1(0)$ \\
\hline 16 & $4(0.34)$ & $4(60)$ & $1(0)$ & $3(0.8)$ & $2(0.4)$ \\
\hline
\end{tabular}


Table 6. Experimental results

\begin{tabular}{|c|c|c|c|c|c|}
\hline $\begin{array}{l}\text { No. of } \\
\text { specimens }\end{array}$ & $\begin{array}{c}7 d \text { compressive } \\
\text { strength/MPa }\end{array}$ & $\begin{array}{c}7 d \text { split- } \\
\text { ting tensile } \\
\text { strength/MPa }\end{array}$ & $\begin{array}{c}\text { 28d compres- } \\
\text { sive strength/ } \\
\text { MPa }\end{array}$ & $\begin{array}{c}\text { 28d split- } \\
\text { ting tensile } \\
\text { strength/MPa }\end{array}$ & $\begin{array}{c}\text { 28d flexural } \\
\text { strength/MPa }\end{array}$ \\
\hline 1 & 59.47 & 12.33 & 81.58 & 15.61 & 13.19 \\
\hline 2 & 69.89 & 26.71 & 94.07 & 23.45 & 14.27 \\
\hline 3 & 56.21 & 21.49 & 75.08 & 29.84 & 20.86 \\
\hline 4 & 33.42 & 25.69 & 52.87 & 24.46 & 17.25 \\
\hline 5 & 75.04 & 32.14 & 87.43 & 37.67 & 24.54 \\
\hline 6 & 56.58 & 19.24 & 71.85 & 20.25 & 22.9 \\
\hline 7 & 46.06 & 15.18 & 60.67 & 18.01 & 7.60 \\
\hline 8 & 41.73 & 12.11 & 47.55 & 8.58 & 5.24 \\
\hline 9 & 51.79 & 18.29 & 61.16 & 20.92 & 15.66 \\
\hline 10 & 42.41 & 13.15 & 55.26 & 14.86 & 10.16 \\
\hline 11 & 40.11 & 14.76 & 56.07 & 27.73 & 15.47 \\
\hline 12 & 23.82 & 8.21 & 36.17 & 14.03 & 5.99 \\
\hline 13 & 44.45 & 14.70 & 55.08 & 16.81 & 9.48 \\
\hline 14 & 34.46 & 13.77 & 53.92 & 20.97 & 11.15 \\
\hline 15 & 28.55 & 9.81 & 39.88 & 13.65 & 13.83 \\
\hline 16 & 23.38 & 7.95 & 36.18 & 11.75 & 10.02 \\
\hline
\end{tabular}

solid. Strip mould after $24 \mathrm{~h}$, being cured to $7 \mathrm{~d}$, 28d. After curing, dry the test piece and conduct mechanical property experiment.

Measure failure load on universal testing machine, calculating compressive strength, splitting tensile strength, flexural strength according to corresponding equation respectively.

The compressive strength was measured by the $70.7 \mathrm{~mm} \times 70.7 \mathrm{~mm} \times 70.7 \mathrm{~mm}$ cube specimens, the tensile strength was measured by the $100 \mathrm{~mm} \times 100 \mathrm{~mm} \times 100 \mathrm{~mm}$ cube specimens, and the flexural strength was measured by $40 \mathrm{~mm} \times 40 \mathrm{~mm} \times 160 \mathrm{~mm}$ prism specimens.

\section{Experimental result and analysis}

Experimental results of $7 \mathrm{~d}, 28 \mathrm{~d}$ compressive strength and splitting tensile strength and $28 \mathrm{~d}$ flexural strength are shown in Table 6. The purpose of this experiment is to study the effect of individual factors on the properties of ECC, and the combination of the interaction between the factors is complex, so the interaction between factors is not discussed in this paper.

\subsection{Intuitive analysis}

It is known from No. 3 and No.7 28d compressive strength in Table 6 that:

When water-binder ratio is the lowest $(0.19)$, fly ash substitution rate is $45 \%$, when using high desert sand substitute $(60 \%)$, we can still prepare high strength ECC with compressive strength above $75 \mathrm{Mpa}$ (No. 3); when waterbinder ratio is low (0.24), even fly ash substitution rate is up to $45 \%$, using extremely high desert sand substitution rate (90\%), the compressive strength is still up to $60 \mathrm{Mpa}$ (No. 7).

\subsection{Range analysis}

Range analysis is shown in Table 7.

The influence trends of each factor in the strength is shown in Figure 1.

It is known from Table 7 and Figure 1 that:

(1) As water-binder ratio increases, compressive strength, splitting tensile strength and flexural strength show decreasing trend; increase of the fly ash substitution rate will result in a decrease of 3 properties. As desert sand substitution rate increases, compressive strength and splitting tensile strength both increase first and decrease slowly then. Flexural strength shows the trend of slow decrease. When the desert sand substitution rate is $30 \%$, compressive strength and splitting tensile strength reach the highest; as Proportion of PVA fiber increases, compressive strength and splitting tensile strength increases first and decreases then, when the proportion is $0.8 \%$, compressive strength and splitting tensile strength reach the highest; flexural strength increases with progressive increase of proportion of PVA fiber. As a proportion of steel fiber increases, compressive strength increases first and de- 

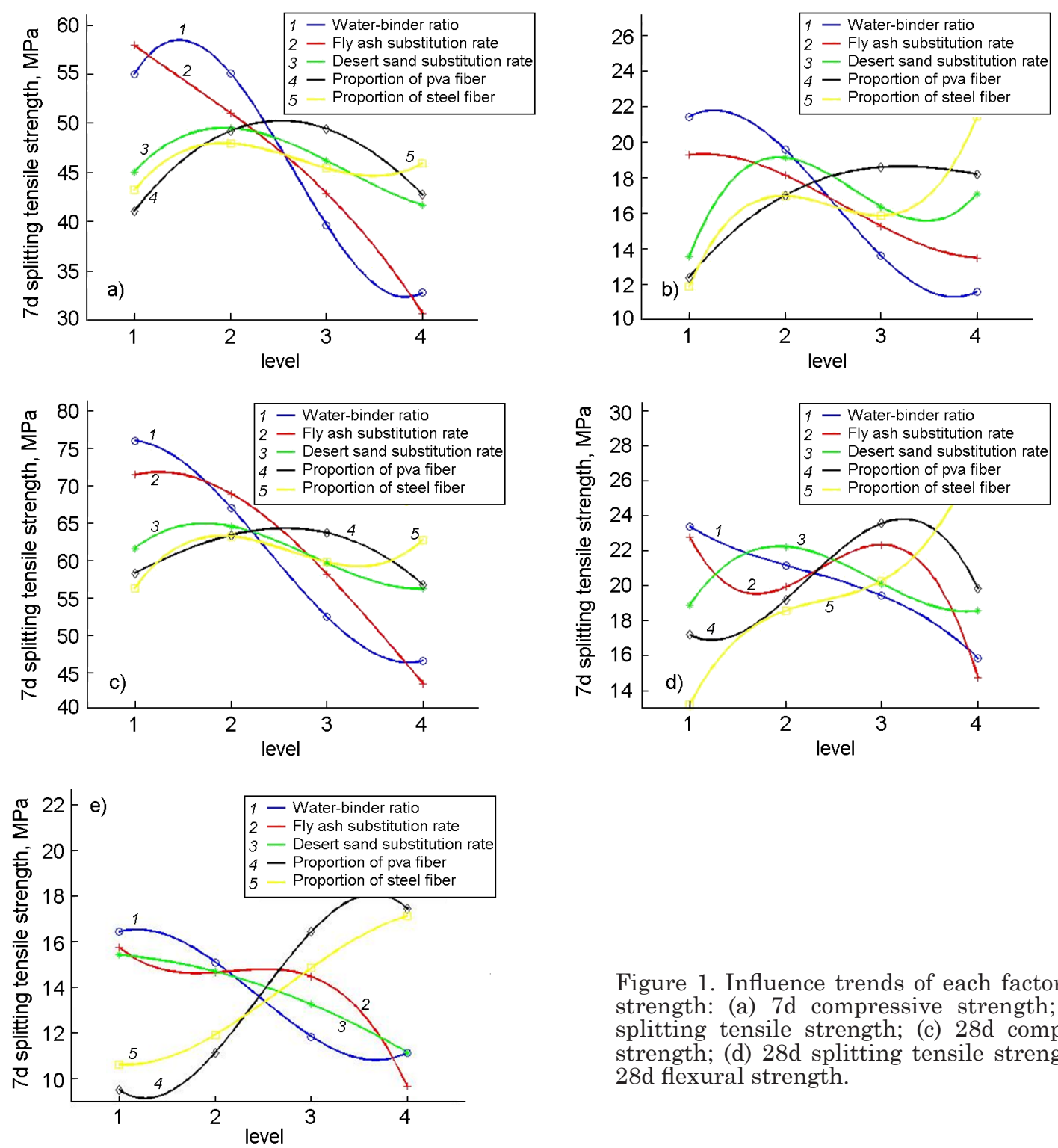

creases then, splitting tensile strength and flexural strength show an increasing trend.

(2) For $7 d$ compressive strength, ranking of every influencing factors is: fly ash substitution rate $(\mathrm{B})>$ water-binder ratio $(\mathrm{A})>$ Proportion of PVA fiber(D) > desert sand substitute (C) > Proportion of steel fiber(E), the better combination of condition is A2B1C2D3E2. For 28d compressive strength, ranking of every influencing factors is: water-binder ratio (A) > fly ash substitution rate $($ B) $>$ desert sand substitute $($ C) $>$ Proportion of PVA fiber(D) >Proportion of steel fiber(E), the better combination of condition is A1B1C2D3E2. For 7d splitting tensile strength, ranking of every influencing factors is: waterbinder ratio (A) > Proportion of steel fiber(E) > Proportion of PVA fiber(D) $>$ fly ash substitution rate $(B)>$ desert sand substitute $(\mathrm{C})$, the better combination of condition is A1B1C2D3E4.

Figure 1. Influence trends of each factor in the strength: (a) 7d compressive strength; (b) 7d splitting tensile strength; (c) 28d compressive strength; (d) 28d splitting tensile strength; (e) $28 \mathrm{~d}$ flexural strength.

For 28d splitting tensile strength, ranking of every influencing factors is: Proportion of steel fiber(E) $>$ fly ash substitution rate $(\mathrm{B})>$ waterbinder ratio (A) $>$ Proportion of PVA fiber(D) $>$ desert sand substitute (C), the better combination of condition is A1B1C2D3E4. For 28d flexural strength, ranking of every influencing factors is: Proportion of PVA fiber(D) > Proportion of steel fiber(E) $>$ fly ash substitution rate (B)> water-binder ratio (A) > desert sand substitute $(\mathrm{C})$, the better combination of condition is A1B1C1D4E4.

(3) The influence of water-binder ratio and fly ash substitution rate of compressive strength is especially significant; $7 \mathrm{~d}$ splitting tensile strength is influenced by water-binder ratio most; 28d splitting tensile strength is influenced by Proportion of steel fiber most; 
Table 7. Range analysis

\begin{tabular}{|c|c|c|c|c|c|c|}
\hline Factors & & $\begin{array}{l}\text { A. } \\
\text { Water-binder } \\
\text { ratio }\end{array}$ & $\begin{array}{l}\text { B. } \\
\text { Fly ash substi- } \\
\text { tution rate/\% }\end{array}$ & $\begin{array}{c}\mathrm{C} . \\
\text { Desert sand } \\
\text { substitution } \\
\text { rate/\% }\end{array}$ & $\begin{array}{c}\text { D. } \\
\text { Proportion of } \\
\text { pva fiber } \%\end{array}$ & $\begin{array}{c}\text { E. } \\
\text { Proportion of } \\
\text { steel fiber/\% }\end{array}$ \\
\hline \multirow{5}{*}{$\begin{array}{l}\text { 7d compressive } \\
\text { strength }\end{array}$} & $\mathrm{K}_{1}$ & 54.75 & 57.69 & 44.89 & 40.95 & 43.04 \\
\hline & $\mathrm{K}_{2}$ & 54.85 & 50.84 & 49.33 & 49.05 & 47.78 \\
\hline & $\mathrm{K}_{3}$ & 39.53 & 42.73 & 46.05 & 49.26 & 45.27 \\
\hline & $\mathrm{K}_{4}$ & 32.71 & 30.59 & 41.59 & 42.59 & 45.76 \\
\hline & $\mathrm{R}$ & 22.14 & 27.10 & 7.74 & 8.31 & 4.74 \\
\hline \multirow{5}{*}{$\begin{array}{l}\text { 7d splitting ten- } \\
\text { sile strength }\end{array}$} & $\mathrm{K}_{1}$ & 21.56 & 19.37 & 13.57 & 12.37 & 11.85 \\
\hline & $\mathrm{K}_{2}$ & 19.67 & 18.22 & 19.22 & 17.07 & 17.03 \\
\hline & $\mathrm{K}_{3}$ & 13.60 & 15.31 & 16.42 & 18.68 & 15.91 \\
\hline & $\mathrm{K}_{4}$ & 11.56 & 13.49 & 17.18 & 18.26 & 21.59 \\
\hline & $\mathrm{R}$ & 10.00 & 5.88 & 5.65 & 6.31 & 9.74 \\
\hline \multirow{5}{*}{$\begin{array}{l}28 \mathrm{~d} \text { compres- } \\
\text { sive strength }\end{array}$} & $\mathrm{K}_{1}$ & 75.90 & 71.31 & 61.42 & 58.09 & 56.07 \\
\hline & $\mathrm{K}_{2}$ & 66.88 & 68.78 & 64.39 & 63.19 & 63.02 \\
\hline & $\mathrm{K}_{3}$ & 52.17 & 57.93 & 59.43 & 63.49 & 59.55 \\
\hline & $\mathrm{K}_{4}$ & 46.27 & 43.19 & 55.97 & 56.44 & 62.57 \\
\hline & $\mathrm{R}$ & 29.63 & 28.12 & 8.42 & 7.05 & 6.95 \\
\hline \multirow{5}{*}{$\begin{array}{l}28 \mathrm{~d} \text { splitting } \\
\text { tensile strength }\end{array}$} & $\mathrm{K}_{1}$ & 23.34 & 22.75 & 18.84 & 17.16 & 13.18 \\
\hline & $\mathrm{K}_{2}$ & 21.13 & 19.88 & 22.20 & 19.14 & 18.53 \\
\hline & $\mathrm{K}_{3}$ & 19.39 & 22.31 & 20.08 & 23.53 & 20.23 \\
\hline & $\mathrm{K}_{4}$ & 15.80 & 14.71 & 18.54 & 19.82 & 27.71 \\
\hline & $\mathrm{R}$ & 7.54 & 8.04 & 3.66 & 6.37 & 14.53 \\
\hline \multirow{5}{*}{$\begin{array}{l}\text { 28d flexural } \\
\text { strength }\end{array}$} & $\mathrm{K}_{1}$ & 16.39 & 15.72 & 15.40 & 9.48 & 10.61 \\
\hline & $\mathrm{K}_{2}$ & 15.07 & 14.62 & 14.66 & 11.12 & 11.89 \\
\hline & $\mathrm{K}_{3}$ & 11.82 & 14.44 & 13.23 & 16.40 & 14.81 \\
\hline & $\mathrm{K}_{4}$ & 11.12 & 9.63 & 11.12 & 17.41 & 17.10 \\
\hline & $\mathrm{R}$ & 5.27 & 6.09 & 4.28 & 7.93 & 6.49 \\
\hline
\end{tabular}

Proportion of PVA fiber influences flexural strength most.

(4) According to integrated balance method, better combination of conditions is determined as A1B1C2D3E4.

\subsection{Variance analysis}

Variance analysis is shown in Table 8. Taking into account the four-level and five-factor orthogonal test, in the variance analysis, the factor whose mean square is the smallest is considered as error to calculate the value of $\mathrm{F}$.

It is known from Table 8 that:

-According to mean square value, rank significance of influence of every factor on mechanical property from large to small, for splitting tensile strength, flexural strength, $7 \mathrm{~d}$ compressive strength, the ranking is in accordance with range analysis; for $28 \mathrm{~d}$ compressive strength, ranking of influence of every factors is: waterbinder ratio (A) >fly ash substitution rate (B)
$>$ Proportion of PVA fiber(D) > desert sand substitute (C) > Proportion of steel fiber(E), slightly different from range analysis.

- Water-binder ratio (A) and fly ash substitution rate $(\mathrm{B})$ influence $7 \mathrm{~d}$ compressive strength highly significantly $(\alpha=0.01)$, influence $28 \mathrm{~d}$ compressive strength moderately significant ( $\alpha$ $=0.05)$; Proportion of steel fiber(E) influences 28d splitting tensile strength significantly. Variation of desert sand substitute (C) does not influence every performance obviously.

\section{Failure modes of specimens}

\subsection{Compression failure}

The specimen without fiber often breaks unexpectedly, with the larger piece falling off as shown in Figure 2 (a). The specimen with only steel fiber has small piece falling off, with relatively complete contour, the surface shows several cracks with certain width, as shown in 
Che Jialing et al. / Experimental research on mechanical properties ...

Table 8. Variance analysis

\begin{tabular}{|c|c|c|c|c|c|c|}
\hline Properties & $\begin{array}{c}\text { Variance } \\
\text { origin }\end{array}$ & Square Sum & $\begin{array}{l}\text { degree of } \\
\text { freedom }\end{array}$ & $\begin{array}{c}\text { Mean } \\
\text { square }\end{array}$ & $\mathrm{F}$ & Critical value \\
\hline \multirow{7}{*}{$\begin{array}{l}7 \mathrm{~d} \text { com- } \\
\text { pressive } \\
\text { strength }\end{array}$} & $\mathrm{A}$ & $\mathrm{SA}=1488.698$ & 3 & 496.233 & $32.746^{* * *}$ & F0.01 $(3,3)=29.5$ \\
\hline & $\mathrm{B}$ & $\mathrm{SB}=1628.132$ & 3 & 542.711 & $35.814^{* * *}$ & F0.05 $(3,3)=9.3$ \\
\hline & $\mathrm{C}$ & $\mathrm{SC}=122.519$ & 3 & 40.840 & 2.695 & $\mathrm{~F} 0.1(3,3)=5.4$ \\
\hline & $\mathrm{D}$ & $\mathrm{SD}=223.502$ & 3 & 74.501 & 4.916 & - \\
\hline & $\mathrm{E}$ & $\mathrm{SE}=45.461$ & 3 & 15.154 & 1.000 & - \\
\hline & Error & $\mathrm{Se}=45.461$ & 3 & 15.154 & - & - \\
\hline & Total & $\mathrm{ST}=3508.311$ & 15 & - & - & - \\
\hline \multirow{7}{*}{$\begin{array}{l}\text { 7d split- } \\
\text { ting tensile } \\
\text { strength }\end{array}$} & $\mathrm{A}$ & $\mathrm{SA}=273.493$ & 3 & 91.164 & 4.168 & F0.01 $(3,3)=29.5$ \\
\hline & $\mathrm{B}$ & $\mathrm{SB}=86.391$ & 3 & 28.797 & 1.317 & F0.05 $(3,3)=9.3$ \\
\hline & $\mathrm{C}$ & $\mathrm{SC}=65.611$ & 3 & 21.870 & 1.000 & $\mathrm{~F} 0.1(3,3)=5.4$ \\
\hline & $\mathrm{D}$ & $\mathrm{SD}=100.707$ & 3 & 33.569 & 1.535 & - \\
\hline & $\mathrm{E}$ & $\mathrm{SE}=192.503$ & 3 & 64.168 & 2.934 & - \\
\hline & Error & $\mathrm{Se}=65.611$ & 3 & 21.870 & - & - \\
\hline & Total & $\mathrm{ST}=718.704$ & 15 & - & - & - \\
\hline \multirow{7}{*}{$\begin{array}{l}28 \mathrm{~d} \text { com- } \\
\text { pressive } \\
\text { strength }\end{array}$} & $\mathrm{A}$ & $\mathrm{SA}=2199.000$ & 3 & 733.000 & $17.707 * *$ & F0.01 $(3,3)=29.5$ \\
\hline & $\mathrm{B}$ & $\mathrm{SB}=1965.632$ & 3 & 655.211 & $15.828^{* *}$ & F0.05 $(3,3)=9.3$ \\
\hline & $\mathrm{C}$ & $\mathrm{SC}=149.889$ & 3 & 49.963 & 1.207 & $\mathrm{~F} 0.1(3,3)=5.4$ \\
\hline & $\mathrm{D}$ & $\mathrm{SD}=153.330$ & 3 & 51.110 & 1.235 & - \\
\hline & $\mathrm{E}$ & $\mathrm{SE}=124.187$ & 3 & 41.396 & 1.000 & - \\
\hline & Error & $\mathrm{Se}=124.187$ & 3 & 41.396 & - & - \\
\hline & Total & $\mathrm{ST}=4592.038$ & 15 & - & - & - \\
\hline \multirow{7}{*}{$\begin{array}{l}\text { 28d split- } \\
\text { ting tensile } \\
\text { strength }\end{array}$} & $\mathrm{A}$ & $\mathrm{SA}=121.824$ & 3 & 40.608 & 3.661 & F0.01 $(3,3)=29.5$ \\
\hline & $\mathrm{B}$ & $\mathrm{SB}=163.682$ & 3 & 54.561 & 4.919 & F0.05 $(3,3)=9.3$ \\
\hline & $\mathrm{C}$ & $\mathrm{SC}=33.274$ & 3 & 11.091 & 1.000 & $\mathrm{~F} 0.1(3,3)=5.4$ \\
\hline & $\mathrm{D}$ & $\mathrm{SD}=85.166$ & 3 & 28.389 & 2.560 & - \\
\hline & $\mathrm{E}$ & $\mathrm{SE}=432.651$ & 3 & 144.217 & $13.003^{* *}$ & - \\
\hline & Error & $\mathrm{Se}=33.274$ & 3 & 11.091 & - & - \\
\hline & Total & $\mathrm{ST}=836.597$ & 15 & - & - & - \\
\hline \multirow{7}{*}{$\begin{array}{l}\text { 28d flexural } \\
\text { strength }\end{array}$} & $\mathrm{A}$ & $\mathrm{SA}=77.111$ & 3 & 25.704 & 1.816 & F0.01 $(3,3)=29.5$ \\
\hline & $\mathrm{B}$ & $\mathrm{SB}=88.122$ & 3 & 29.374 & 2.075 & F0.05 $(3,3)=9.3$ \\
\hline & $\mathrm{C}$ & $\mathrm{SC}=42.468$ & 3 & 14.156 & 1.000 & $\mathrm{~F} 0.1(3,3)=5.4$ \\
\hline & $\mathrm{D}$ & $\mathrm{SD}=181.829$ & 3 & 60.610 & 4.282 & - \\
\hline & $\mathrm{E}$ & $\mathrm{SE}=102.513$ & 3 & 34.171 & 2.414 & - \\
\hline & Error & $\mathrm{Se}=42.468$ & 3 & 14.156 & - & - \\
\hline & Total & $\mathrm{ST}=492.043$ & 15 & - & - & - \\
\hline
\end{tabular}

NOTE: *** Significant at a level of $0.01 ; * *$ Significant at a level of 0.05 .

Figure 2(b). The specimen with steel-PVA fiber has many micro cracks in the surface, with almost nothing falling off in the surface, keeping good integrity, as shown in Figure 2(c), with cracks marked with marker pen.

\subsection{Splitting failure}

The specimen without fiber will suddenly split with a "Bang" under max load, totally breaking, as shown in Figure 3(a). The specimen with only steel fiber will form several wider main cracks, and the specimen is still linked together, as shown in Figure 3(b). The specimen with steel-PVA fiber forms 1 or two thinner main cracks and several micro cracks, failure mark in the surface is not apparent, as shown in Figure 3(c). 


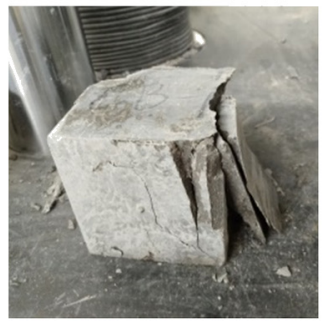

a)

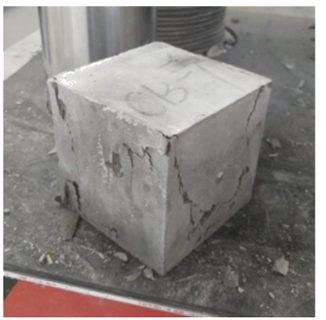

b)

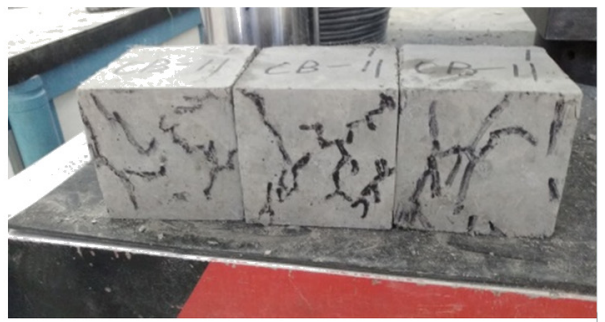

c)

Figure 2. Compression failure modes. (a) Specimen without fiber (b) Specimen with only steel fiber Specimen with steel-PVA fiber.

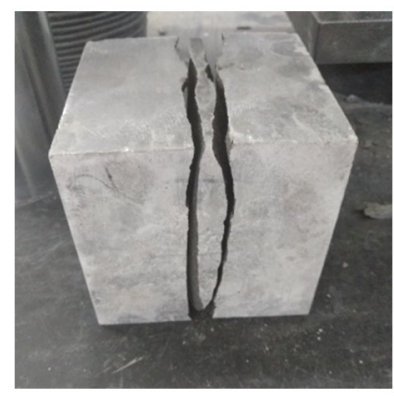

a)

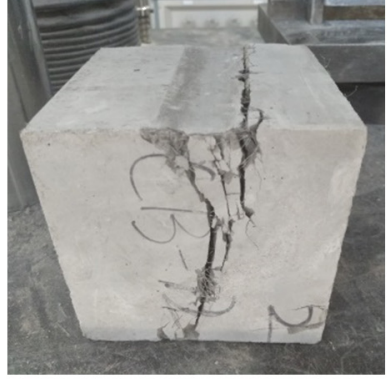

b)

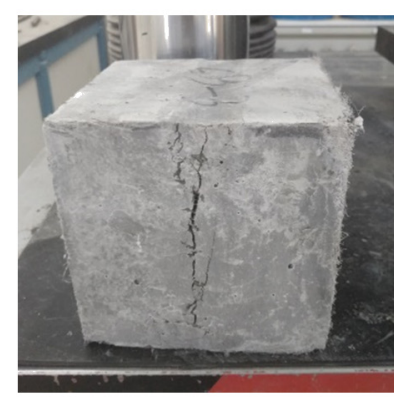

c)

Fig. 3. Splitting failure modes. (a) Specimen without fiber, (b) Specimen with only steel fiber, (c) Specimen with steel-PVA fiber.

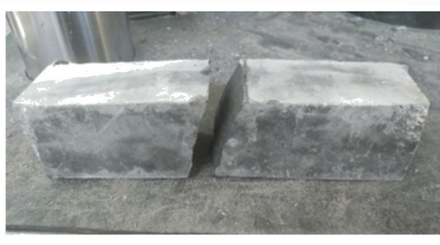

a)

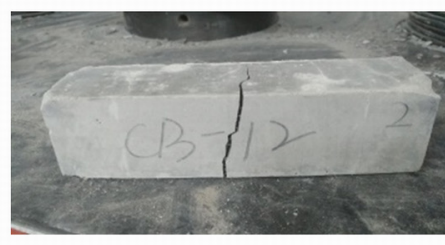

b)

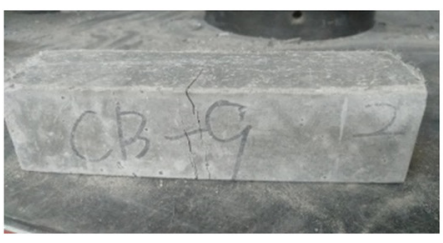

c)

Fig. 4. Flexural failure modes. (a) Specimen without fiber, (b) Specimen with only steel fiber, (c) Specimen with steel-PVA fiber.

\subsection{Flexural failure}

The specimen without fiber gives out crisp sound of breaking when failing, the test piece is bent to two halves totally, as shown in Figure 4(a). The test specimen with only steel fiber forms a wider crack in tensile area, the compressive area is still linked together, as shown in Figure 4(b). The specimen with steel-PVA fiber breaks to smaller cracks in compressive area, with relatively integrated form, as shown in Figure 4(c).

\subsection{Cause analysis}

Test pieces with different type of fiber have different failure modes(There is no significant difference between specimens with only PVA fiber and specimens with steel-PVA fiber, so the failure mode of a specimen with only PVA fiber is not presented in the text). Two kinds of fiber used in the experiment are different greatly in size of the diameter, playing bridging role in different scale. PVA fibers mainly controls micro crack at an early stage of bearing load, steel fiber mainly controls macro crack. The test piece without fiber will generate a fragile burst failure; steel fiber ECC typically produces several wider main cracks when it fails; steelPVA fiber ECC will produce many micro cracks when it fails, the form is damaged less, failure is slow, showing better ductility.

\section{Conclusion}

Using inexhaustible desert sand and industrial waste fly ash, through orthogonal experiment, this paper mainly researches influence trend of 5 factors, including water-binder ratio, fly ash substitution rate, desert sand substitution rate, proportion of PVA fiber, and proportion of steel fiber on ECC strengths, in order to find main factor influencing strength. According to failure mode of test pieces, the role 
played in desert sand ECC by different fiber is analyzed. And it is concluded that:

- When water-binder ratio is low, even if adopting high fly ash substitute and adding large amount of desert sand, high strength ECC can still be prepared, which is of significance for desert sand ECC at key location of high rise anti-seismic structure.

- According to integrated balance method, finalized better condition of factor combination is $\mathrm{A} 1 \mathrm{~B} 1 \mathrm{C} 2 \mathrm{D} 3 \mathrm{E} 4$, namely water-binder ratio is 0.19 , fly ash substitution rate is $15 \%$, the desert sand substitution rate is $30 \%$, proportion of PVA fiber is $0.8 \%$, proportion of steel fiber is $1.2 \%$.

-Water-binder ratio and fly ash substitution rate influence compressive strength highly significant $(\alpha=0.01)$, but low water-binder ratio and fly ash substitution rate result in even larger compressive strength; proportion of steel fibers significantly $(\alpha=0.05)$ influences 28d splitting tensile strength, and steel fiber is conducive to development of splitting tensile strength; PVA fiber is conducive to the development of flexural strength.

- Desert sand substitution rate does not significantly influence every mechanical properties, but appropriate desert sand substitution rate can improve ECC property to a certain degree. In addition, max desert sand substitute in the experiment has been up to $90 \%$, and the experimental group adopting $90 \%$ desert sand substitution rate does not decrease obviously in compressive strength, splitting tensile strength compared with the experimental group without desert sand, but flexural strength decreases to some extent. So it is inferred that as raw material of ECC, desert sand can further totally substitute for river sand.

- PVA fiber enhances ductility of desert sand ECC, making the failure process of test piece failure intend to slow, conducive to multicrack development of failure.

\section{Acknowledgements}

This work was financially supported by the National Natural Science Foundation of China with No. 51408328.

\section{References}

1. V. C. Li, D.K. Mishra. A.E. Naaman, et al, $A d v$. Cement Based Mater., 1, 142, 1994.

2. V. C. Li, J. Adv. Conc. Techn., 1, 215,2003.

3. S. F. U. Ahmed, M. Maalej, P. Paramasivam, J. Ferrocement, 33, 172, 2003.

4. S. F. U. Ahmed, M. Maalej, P. Paramasivam, Constr. Building Mater., 21, 1088, 2007.

5. G. Fischer, V. C. Li, Struct. J., 99, 781, 2002

6. Xu Shilang, Cai Xinhua, Acta Mater. Compos. Sinica, 27, 177, 2010.

7. Y. Zhu, Y. Yang, Y. Yao, Constr. Building Mater., 36, 1076, 2012.

8. H. Zhang, M.D. Lepech, G.A. Keoleian, et al, J. Infrastruct. Syst., 16, 299, 2009.

9. M. Şahmaran, V. C. Li, Structures Congress 2009: Don't Mess with Structural Engineers: Expanding Our Role., 1-13, 2009.

10. M.D. Lepech, V. C. Li, Mater. Struct., 42, 1185, 2009.

11. V. C. Li, Int. J.Concrete Struct.Mater., 6, 135, 2012.

12. K. Sirijaroonchai, S. El-Tawil, G. Parra-Montesinos, Cement Concrete Comp, 32, 62, 2010.

13. M. Li, V.C. Li, Mater. Struct., 46, 405, 2013.

14. Z. Zhang, S. Qian, H. Ma, Constr.Building Mater., 52, 17, 2014 . 